Trauma Berufskrankh 2005 · 7 [Suppl 2]: S321-S326 DOI 10.1007/s10039-005-1050-6

Online publiziert: 14. September 2005

(c) Springer Medizin Verlag 2005

R. Beisse · BG-Unfallklinik, Murnau

\title{
Komplikationen in der endoskopischen Wirbelsäulenchirurgie
}

Weise Tumoren und Metastasen $[5,12]$ entfernt werden.

\section{Besonderheiten der endoskopischen Operationstechnik}

Aufgrund der zentralen Einbettung der Wirbelsäule in unmittelbarer Nähe zu den großen Gefäßen und dem ZNS (• Abb. 2) setzt die Wirbelsäulenchirurgie in besonderem Maß einen sicheren und handwerklich einwandfreien Umgang mit Instrumenten und Implantaten voraus. Bei der Umsetzung der Erfahrungen aus der offenen Wirbelsäulenchirurgie in das endoskopische Verfahren gilt es, sich einige Punkte deutlich vor Augen zu führen:

- Die für das endoskopische Verfahren typische Dissoziation von optischer Wahrnehmung und Ort der manueller Tätigkeit führt zu einem Gefühl des vermeintlichen Abstands vom operativen Geschehen und zu einem falschen Gefühl der Sicherheit.

- Die Bilddarstellung ist zweidimensional und erschwert eine exakte Winkelund Tiefeneinschätzung.

- Beim Setzen von Schrauben und Spickdrähten ist man in erhöhtem Maß auf eine subtile fluoroskopische Darstellung angewiesen, bei der keine Kompromisse hinsichtlich der Genauigkeit der Projektion der Wirbelkörper eingegangen werden dürfen.

- Das endoskopische Verfahren steht und fällt mit der Qualität des Bildübertragungssystems, d. h. die Verwendung einer hoch auflösenden (3-
Chip)-Kamera und lichtstarken (Xenon-)Lichtquelle ist dringend zu empfehlen. Abstriche an der optischen Qualität führen zu suboptimalen operativen Ergebnissen.

- Das Gefährdungspotenzial von Instrumenten und Anwendungen im offenen oder endoskopischen Einsatz differiert z. T. erheblich, z. B. bei der Anwendung von Gefäßclips (• Abb. 3), monopolarem Strom (• Abb. 4) und dem Einsatz von Fräsen.

\section{Technische Ausstattung [3]}

Für den Zugang benutzen wir 4 mehrfach verwendbare flexible Trokare mit Gewinde mit einem Durchmesser von $11 \mathrm{~mm}$. Diese sind flexibel, um den Druck auf das interkostale Gefäß-Nerven-Bündel möglichst gering zu halten. Lichtreflexionen und dadurch bedingte Störungen der Lichtstärkeregulation der Lichtquelle können durch die Verwendung von schwarzen Trokaren und von Instrumenten mit matter Oberfläche vermieden werden.

Die Ausleuchtung der Thoraxhöhle erfordert eine lichtstarke Xenonquelle. Für die Bildübertragung verwenden wir eine $30^{\circ}$-Kamera, die eine Positionierung fern$\mathrm{ab}$ des Arbeitsportals und damit ein störungsfreies Arbeiten sowie eine variable Einstellung des Sichtwinkels ermöglicht. Der intraoperative Situs wird auf 2 Flachbildschirme übertragen, die Teil eines so genannten Endoskopieturms sind, auf dem sich eine digitale Bildaufzeichnungseinheit und der Generator für das Ultraschallmesser befinden. Eine Luftinsufflation ist nicht erforderlich. 


\section{Ventrale Instrumentation BWS/LWS}

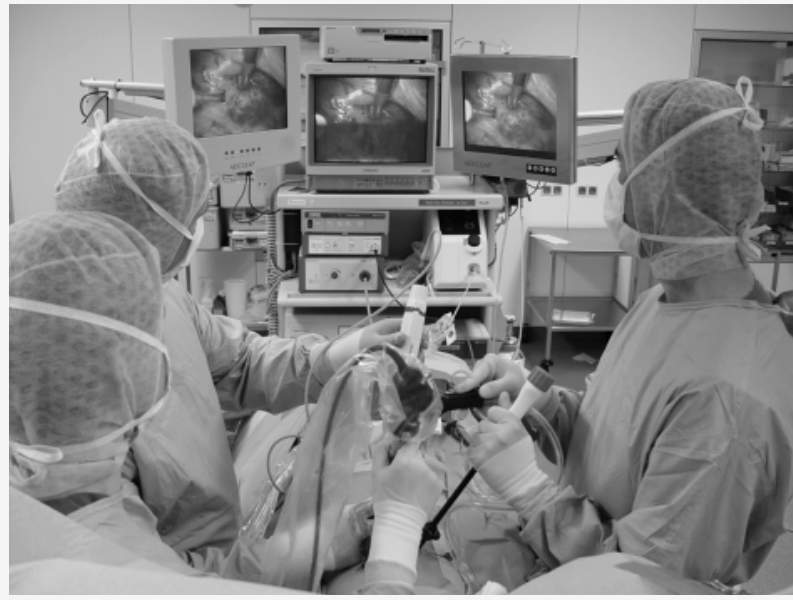

Abb. $1 \Delta$ Endoskopischer Eingriff an der Wirbelsäule

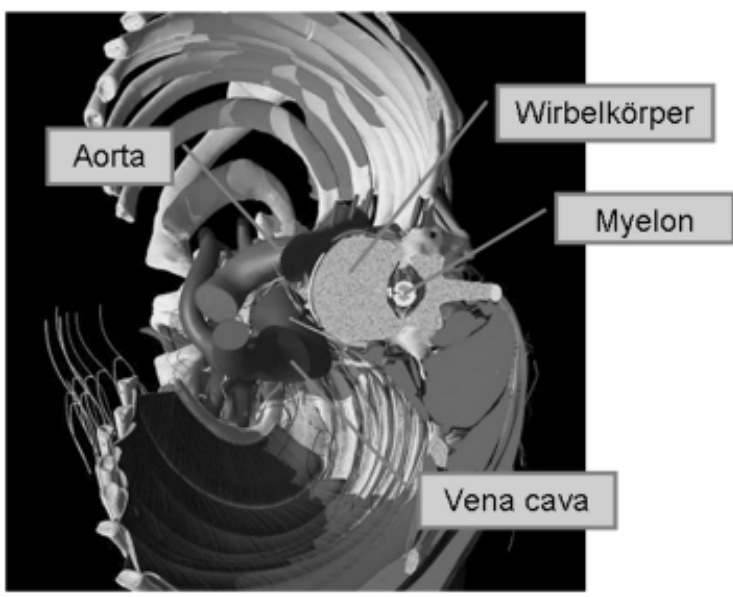

Abb. $2 \Delta$ Topographische Beziehung von Wirbelsäule, Spinalkanal und großen Gefäßen auf Höhe des thorakolumbalen Übergangs

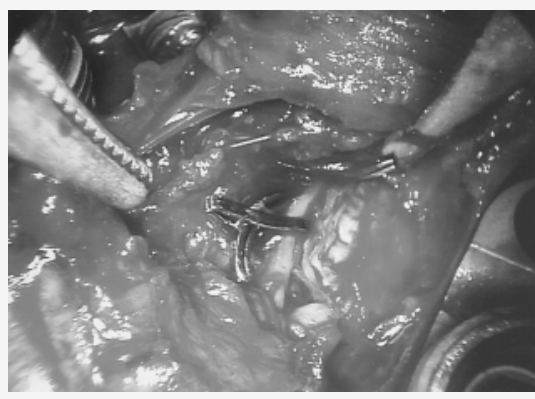

Abb. $3 \Delta$ Blutungsgefahr durch falsches Setzen von Gefäßclips

Für die Weichteil- und Knochenpräparation werden heute von verschiedenen Herstellern Sets von Instrumenten angeboten, bei denen man auf eine beiderseitige Skalierung zur Tiefenbestimmung sowie auf eine ergonomische Führung und Bedienung achten sollte.

\section{Komplikationen und Komplikationsmanagement}

Die von Aebi [1] eingeführte Typologie der Komplikationen gilt prinzipiell auch für die endoskopische Wirbelsäulenchirurgie. Sie werden verursacht durch:

$$
\begin{aligned}
& \text { - Reposition } \\
& \text { - Zugang } \\
& \text { - Präparation } \\
& \text { - Setzen von Schrauben und Implantaten } \\
& \text { - das Implantat selbst } \\
& \text { - mangelnde Fusion } \\
& \text { - Infekt }
\end{aligned}
$$

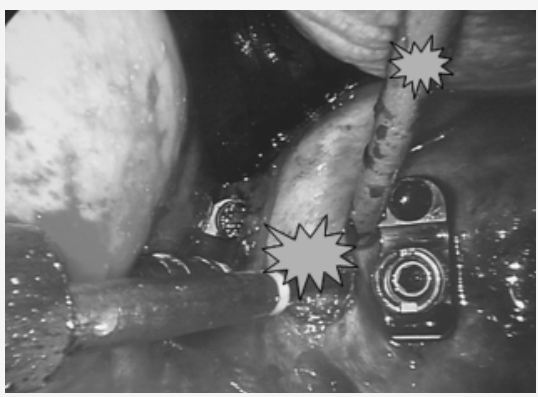

Abb. $4 \triangle$ Gefahr der Verbrennung durch Anwendung monopolaren Stroms

\section{Reposition}

Sie ist bei größeren Fehlstellungen über die ventrale Säule allein aus biomechanischen Gründen limitiert. Komplikationen treten durch die inadäquate Einbringung von Kräften an Implantaten oder Endplatten im Rahmen von Repositionsversuchen auf und können zur primären oder vorzeitigen Auslockerung von Implantaten führen.

\section{Zugang und Präparation}

Von größerer Bedeutung für das endoskopische Vorgehen, insbesondere am thorakolumbalen Übergang, sind die zugangsbedingten Komplikationen. Die Nähe des Zwerchfells (• Abb. 5) und der unmittelbar darunter befindlichen Organe erfordern große Umsicht beim Setzen der Trokare. Wir empfehlen, das am meisten kranial in der Thoraxhöhle gelegene Portal für die Op-

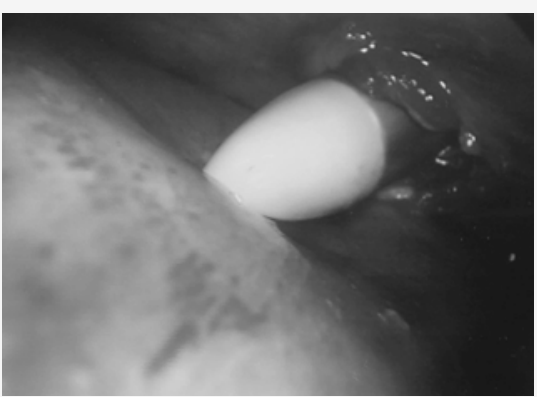

Abb. $5 \Delta$ Gefahr der Zwerchfellperforation durch Instrumente am thorakolumbalen Übergang

tik zuerst anzulegen und den Thorax über eine kleine Thorakotomie von $2 \mathrm{~cm}$ zu eröffnen, die es gestattet, den Situs digital auszutasten, um Adhäsionen von Lunge, Pleura und Zwerchfell auszuschließen. Die weiteren Portale können dann unter endoskopischer Kontrolle angelegt werden.

Die Operation sollte mit einem Rundumblick über die Thoraxhöhle beginnen und enden. Vor Entfernung der Optik muss unbedingt die vollständige Entfaltung der Lunge endoskopisch kontrolliert werden, um Atelektasen und Ergussbildung vorzubeugen. Zur Vorbeugung von Interkostalneuralgien empfehlen wir die Verwendung flexibler Trokare, die den Druck auf das interkostale Gefäß-Nerven-Bündel auf ein nicht vermeidbares Minimum reduzieren.

Durch den minimalinvasiven Zugang und die konsequente Umsetzung dieser Empfehlungen konnte die Rate zugangsbedingter Komplikationen im Verlauf erheblich gesenkt werden. Laut einer Analy- 
se unserer ersten 371 Patienten betrug sie $5,4 \%$. Die diesbezügliche Rate bei offenen Eingriffen lag in einer Multicenterstudie von Faciszewski et al. [6] noch bei knapp über $14 \%$ und wurde von Knop et al. [9] auf $15 \%$ beziffert.

\section{Verletzungen von Gefäßen}

Das Komplikationspotenzial wirbelsäulenchirurgischer Eingriffe muss aufgrund der unmittelbaren Nähe lebenswichtiger Strukturen als hoch angesehen werden. Während das Risiko neurologischer Komplikationen wegen des auf Höhe des thorakolumbalen Übergangs auslaufenden Rückenmarks und der größeren Weite des Spinalkanals gegenüber der Brustwirbelsäule eher als geringer zu erachten ist, gilt ein umso größeres Augenmerk den ventral gelegenen Gefäßen. Deren Verletzung stellt eine lebensbedrohliche Komplikation dar, deren Beherrschung eine unverzügliche Thorakophrenotomie mit großzügiger Freilegung, Mobilisation und Anschlingen des Gefäßes erfordert. Die zahlreichen Abgänge aus der Aorta (Truncus coeliacus, A. mesenterica superior und Aa. renales) und Kollateralen auf der Höhe des thorakolumbalen Übergangs erschweren die zur Versorgung des Lecks erforderliche Bluttrockenheit ganz erheblich.

Zur Vermeidung derartiger Komplikationen ist eine absolute handwerkliche Vertrautheit des Operateurs mit dem von ihm verwendeten Instrumentarium und mit den Besonderheiten und Schwächen der endoskopischen Technik zu fordern. Bei der Einbringung des Wirbelkörperersatzes und dem Eindrehen von Schrauben kann durch die Verwendung des Bildwandlers eine bedrohliche Fehllage frühzeitig erkannt und dieser vorgebeugt werden.

In unserem Klientel von über $500 \mathrm{~Pa}$ tienten in den ersten 6 Jahren nach Einführung der endoskopischen Technik lag die Rate von Gefäßkomplikationen (Aortenleckage) bei o,4\%. Der erste Fall betraf einen Revisionseingriff ( $\bullet$ Abb. 6a) mit einer atypischen Verlagerung der Aorta und ihrer kurzstreckigen Eröffnung bei Freilegung des gelockerten Implantats. Es folgte die Konversion zur Thorakotomie und die direkte Naht der Aorta ( $\bullet$ Abb. 6b, c).
Der weitere operative und postoperative Verlauf war komplikationslos. Die zweite Verletzung der Aorta trat im Zusammenhang mit der Anwendung einer in der Wirbelsäulenchirurgie gebräuchlichen Hochfrequenzfräse auf. Bei Durchführung der letzten Frässchritte zur Teilkorporektomie auf Höhe BWK 6/7 kam es zur der Blutung aus der Aorta. Trotz sofortiger Thorakotomie, Freilegung und Ausklemmung der Aorta von der Gegenseite verstarb der Patient im Blutungsschock. Die Obduktion und feingewebliche Aufarbeitung zeigten die Aortenwand kurzstreckig schlitzförmig eröffnet, hervorgerufen am ehesten durch einen in den Fräser involvierten Metallclip oder ein Knochenfragment. Wir haben seitdem konsequent auf die Verwendung von Fräsen verzichtet.

Der Vergleichswert der Rate von Verletzungen großer Gefäße einer amerikanischen Multicenterstudie von 1223 offenen Eingriffen wurde mit o,08\% angegeben. Die Letalität beträgt im eigenen Krankengut bezogen auf 500 Eingriffe 0,2\% gegenüber 0,3\% der Multicenterstudie.

\section{Verletzungen von Organen}

Aufgrund der unmittelbaren Nähe parenchymatöser Organe in Bezug zum Zwerchfell besteht das Risiko, diese zu verletzen. So haben wir über eine Milzverletzung bei einer 52-jährigen Frau zu berichten, bei der in der Endphase des endoskopischen Eingriffs neben der Thoraxdrainage ein so genannter Pleurakath eingebracht wurde, um eine Entfernung des Restergusses nach Ziehen der Thoraxdrainage nach 2-3 Tagen zu ermöglichen. Unter ausschleichender Relaxierung des Patienten kam es zum Hustenreiz mit Hochschnellen des Zwerchfells und durch das Zwerchfell hindurch zur Perforation der Milz mit der eingeführten Hohlschliffnadel. In der nachfolgenden Laparotomie musste die Milz entfernt werden. In der Folgezeit wurde nach diesem Vorfall auf die Einbringung eines Pleurakaths verzichtet. Die Rate der Organkomplikationen liegt damit ebenfalls bei o,2\% bezogen auf 500 Eingriffe.

\section{Neurologische Komplikationen}

Es wurde eine Läsion der Wurzel L1 durch die Anwendung monopolaren Stroms bei Durchführung der Zwerchfellinzision im
Trauma Berufskrankh

2005 7 [ [Suppl 2]: S321-S326

DOI 10.1007/s10039-005-1050-6

(c) Springer Medizin Verlag 2005

\section{R. Beisse}

\section{Komplikationen in der endoskopischen Wirbelsäulenchirurgie}

\section{Zusammenfassung}

Endoskopische Verfahren in der Behandlung des Wirbelsäulentraumas haben sich in den vergangenen 10 Jahren vom optionalen Zusatzeingriff hin zum Standardeingriff in der Behandlung des spinalen Traumas entwickelt. Die neue Zugangstechnik zusammen mit der Verwendung ungewohnter Instrumente und Implantate birgt besondere Risiken und Komplikationsmöglichkeiten, die in gleicher Häufigkeit in der offenen Wirbelsäulenchirurgie auftreten.

\section{Schlüsselwörter}

Spinales Trauma - Endoskopische Wirbelsäulenchirurgie - Komplikationen · Verletzungen der Wirbelsäule

\section{Complications in endoscopic spinal surgery}

\section{Abstract \\ The past 10 years have seen a change in the status of endoscopic techniques in the treatment of spinal traumas: no lon- ger seen as of optional adjuvant interven- tions, endosscopic operations are now re- garded as standard. The new approach technique together with the use of unfa- miliar instruments and implants involves particular risks and complications, howev- er, which are just as frequent as those en- countereed with open spinal surgery.}

\section{Keywords}

Spinal trauma - Endoscopic spinal surgery - Complications - Spinal injuries 

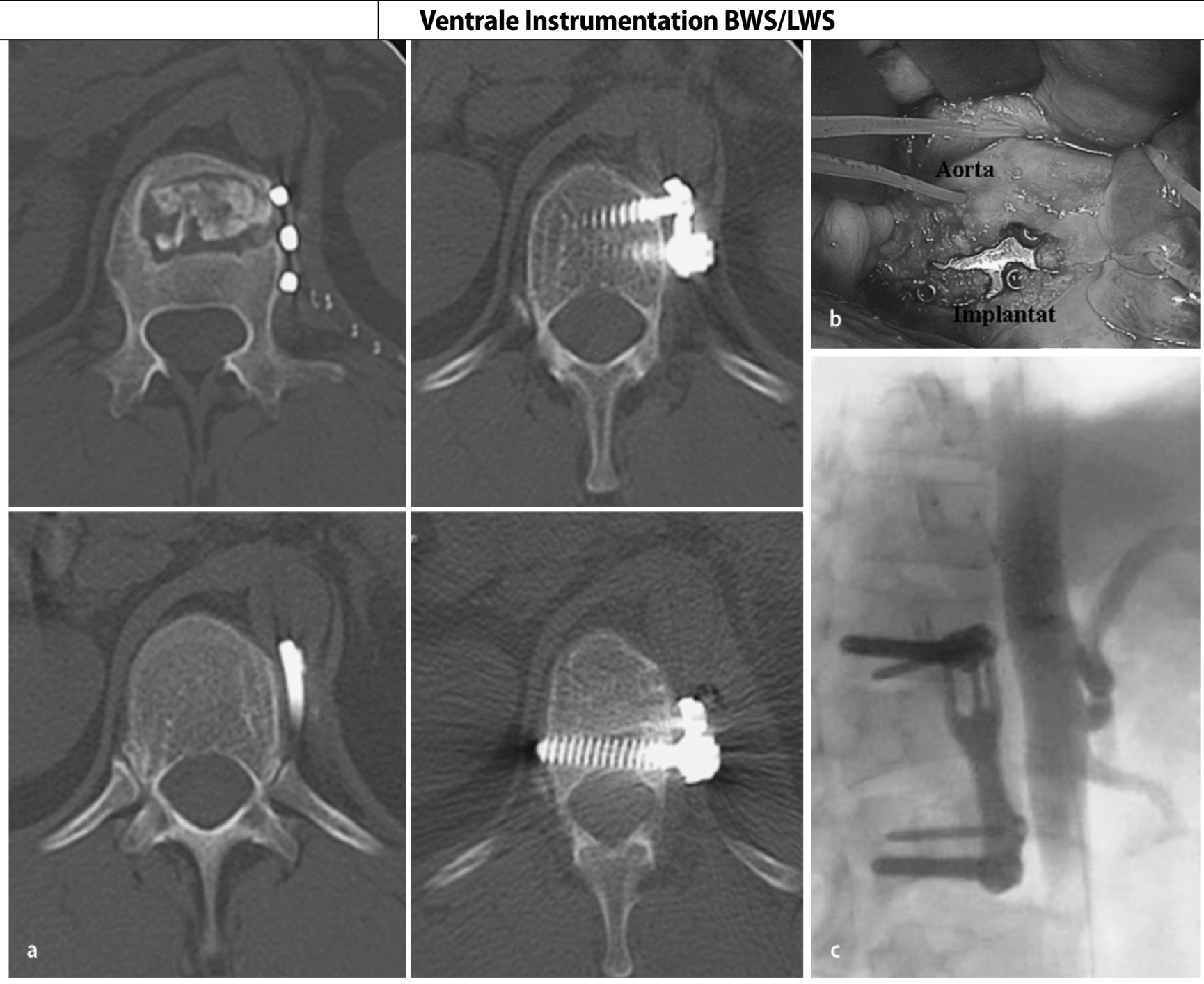

Abb. $6 \Delta$ a Gedeckte Arosion der Aorta bei Implantatlockerung, b Konversion zur Thorakotomie, Ausklemmen der Aorta und Naht, c postoperatives Angiogramm nach Naht der Aorta

Rahmen des Zugangs zum thorakolumbalen Übergang beschrieben. Es handelte sich dabei um die einzige und in ihrer Auswirkung vorübergehende Komplikation im Zusammenhang mit der Inzision des Zwerchfellansatzes beim Zugang zum thorakolumbalen Übergang. Insbesondere war keine diaphragmale Hernienbildung zu verzeichnen. Aus anderen Kliniken sind uns z. T. im Rahmen gutachterlicher Anfragen 2 Fälle von postoperativ aufgetretenen Zwerchfellhernien bekannt geworden, die Anlass geben, das Risiko der Entstehung von diaphragmalen Hernien nach transdiaphragmalem Zugang zum thorakolumbalen Übergang nicht als zu gering einzuschätzen. Wir empfehlen deshalb folgendes Vorgehen [2]:
- Die Inzision sollte nicht radial über der Wirbelsäule, sondern dem Zwerchfellansatz semizirkulär folgend durchgeführt werden. Bei radialer Inzision stehen die Ränder der Lücke durch den intraabdominellen Druck unter höherer Spannung, während bei semizirkulärer Inzision die Ränder aufgrund der Wölbung des Zwerchfells aneinandergepresst werden und leichter verkleben.

- Jede Inzision $>2,5 \mathrm{~cm}$ wird endoskopisch vernäht. Es genügen adaptierende, nicht wasserdichte Nähte. Inzisionen $\leq 2,5 \mathrm{~cm}$, die als Zugang zum 1 . Lendenwirbelkörper ausreichen, verkleben bei korrekter Inzisionsrichtung von selbst.

\section{Komplikationshäufigkeiten}

Komplexe endoskopische Eingriffe sind zwangsläufig mit einem höheren Komplikationsrisiko behaftet. So verzeichneten wir bei $36,7 \%$ (11 von 30 ) der Patienten mit einer anterioren Dekompression [4] eine Komplikation, davon waren allein 5 mit der Entnahme des Beckenkammspans assoziiert und betrafen im Wesentlichen postoperative Schmerzzustände in dieser Region. Die restlichen 6 Komplikationen entfielen auf

- 1 postoperative pulmonale Insuffizienz mit der Notwendigkeit einer prolongierten Beatmung auf der Intensivstation

- 2 interventionsbedürftige pleurale Ergussbildungen 
- 1 Lungenembolie bei tiefer Beinvenenthrombose und präexistenter kompletter Paraplegie

- 1 transiente Irritation des Plexus brachialis, lagerungsbedingt

- 1 persistierende Schädigung des sympathische Nervengeflechts

Aus der Gruppe der Komplikationen durch Implantate, Infekt und ausbleibende knöcherne Fusion ist über ähnliche Ergebnisse endoskopischer und offener Verfahren zu berichten. Wir verzeichneten insgesamt 5 Implantatlockerungen, von denen 4 auf die Z-Platte mit Lockerung einer der vorderen nichtwinkelstabilen Schrauben entfielen. In 1 Fall kam es zur Lockerung des gesamten MACS-Implantats bei $\mathrm{zu}$ weit gestellter Indikation bei schwerer Osteoporose. Von diesen 5 Fällen wurden 3 einem Revisionseingriff wegen des begleitenden Korrekturverlusts unterzogen.

Die Fusionsraten werden zwischen 85 und $90 \%$ angegeben $[7,8]$. Die Vorteile der kleineren Zugänge im Hinblick auf eine mögliche Kontamination des Situs und Infektbildung werden zumindest in der Anfangszeit durch die längeren Operationszeiten des endoskopischen Verfahrens teilweise wettgemacht.

\section{Diskussion}

Die Anfangszeit der endoskopischen Wirbelsäulenchirurgie war gekennzeichnet durch das Fehlen von aufeinander und auf das Verfahren abgestimmten Instrumenten und Implantaten, was sich neben der noch geringeren Erfahrung in längeren Operationszeiten und einer höheren Komplikationsrate niederschlug. Diesbezüglich sind im Verlauf der vergangenen 10 Jahre entscheidende Fortschritte eingetreten, die zu einer kontinuierlichen Verbesserung und höheren Sicherheit des endoskopischen Verfahrens geführt haben.

Wir überblicken an unserer Klinik seit der Einführung der endoskopischen Technik an der Wirbelsäule nunmehr etwa 850 Eingriffe und ein entsprechendes Spektrum an Komplikationen. Deren Schilderung und die Analyse der Ursachen sind zweifellos für ihre Vermeidung in der Zukunft von großer Wichtigkeit. Die Betrachtung der Einzelfälle für sich allein ist allerdings von begrenzter Aussagekraft

Tabelle 1

Komplikationsraten [\%] am vorderen Abschnitt der Wirbelsäule

\begin{tabular}{|lll} 
& Offen [\%] & Endoskopisch [\%] \\
\hline Tiefe Infektion & $0,57-2,0$ & 0,2 \\
\hline Verletzung grosser Gefässe & 0,08 & 0,4 \\
\hline Letalität & 0,3 & 0,2 \\
\hline Neurologisches Defizit & 0,2 & 0,2 \\
\hline Organverletzung & 0 & 0,2 \\
\hline Zugangsmorbidität & 14 & 7,6 \\
• Postthorakotomy-Syndrom & 9,2 & 1,7 \\
• Pleuraerguss & 3,0 & 5,3 \\
• Pneumothorax, persist. & 1,8 & 0,6
\end{tabular}

Nach Faciszewski et al. [6], Sammelstudie, n=1223 Eingriffe

für die Einordnung des endoskopischen Verfahrens im Hinblick auf dessen Risikopotenzial und Komplikationsträchtigkeit. Allein der Vergleich mit der Komplikationsrate offener Verfahren kann hierüber Aufschluss geben. Diesbezüglich werden im Folgenden die Daten einer amerikanischen Multicenterstudie [6] über 1223 Fälle offener Eingriffe am vorderen Abschnitt der Wirbelsäule zum Vergleich herangezogen (• Tabelle 1):

- Die Rate der tiefen Infekte ist beim endoskopischen Vorgehen gegenüber dem offenen Verfahren deutlich geringer und wahrscheinlich Ausdruck der kleinen Zugänge und der geringeren Exposition des Operationssitus mit der damit verbundenen Gefahr der Kontamination und Austrocknung des Operationsfelds.

- Die Rate der Verletzung großer Gefäße und parenchymatöser Organe ist beim endoskopischen Vorgehen gering erhöht. Bei den Organverletzungen entspricht die Rate von 0,2\% einem einzigen Fall!

- Sowohl die Rate von Verletzungen neuraler Strukturen mit neurologischem Defizit als auch die Letalität sind bei beiden Verfahren annähernd gleich.

- Die Rate zugangsbedingter Komplikationen konnte bei Anwendung des endoskopischen Verfahrens nahezu halbiert werden.

Bei der Betrachtung dieses Vergleichs darf nicht vergessen werden, dass hier die Kom- plikationsraten eines seit Jahrzehnten eingeführten (offenen) Verfahrens mit der Häufigkeit von Komplikationen der ersten 500 endoskopischen Eingriffe verglichen werden. Dies gibt Anlass zur Hoffnung, dass bei der zu erwartenden Weiterentwicklung der endoskopischen Technik noch eine Senkung der Komplikationsraten erwartet werden kann.

\section{Fazit}

Endoskopische Verfahren in der Behandlung des Wirbelsäulentraumas haben sich in den vergangenen 10 Jahren von der Bedeutung des optionalen Zusatzeingriffs hin zum Standardeingriff in der Behandlung des spinalen Traumas entwickelt. Sie basieren auf den Prinzipien der klassischen Wirbelsäulenchirurgie der Reposition, Rekonstruktion und Retention des vorderen Abschnitts der Wirbelsäule. Diese Entwicklung wurde durch eine kontinuierliche Verbesserung von Instrumenten begünstigt, die auch die Durchführung komplexer Eingriffe wie eine anteriore Dekompression des Myelons oder die Stellungskorrektur der Wirbelsäule bei posttraumatischer Fehlstellung ermöglichen. Durch die Standardisierung der Operationsschritte und die Weiterentwicklung von Instrumenten und Implantaten liegen die Komplikationsraten endoskopischer Eingriffe im gleichen Bereich wie die offener Verfahren. Die Komplikationen sind Ausdruck der Gefahren und Grenzen des Verfahrens, der Indikationsstellung und des risikobehafteten Umfelds der Wirbelsäulenchirurgie, an dem auch der Einsatz der Endo- 
skopie nichts zu ändern vermag. Die der Einführung endoskopischer Techniken zugrunde liegende Intention einer Senkung der Zugangsmorbidität konnte in vollem Umfang verwirklicht werden.

\section{Korrespondierender Autor}

\section{Prof. Dr. R. Beisse}

BG-Unfallklinik, Prof.-Küntscher-Straße 8, 82418 Murnau

E-Mail: beisse@bgu-murnau.de

Interessenkonflikt: Der korrespondierende Autor versichert, dass keine Verbindungen mit einer Firma, deren Produkt in dem Artikel genannt ist, oder einer Firma, die ein Konkurrenzprodukt vertreibt, bestehen.

\section{Literatur}

1. Aebi M (1989) After-care and rehabilitation following surgical treatment of vertebral fractures. Ther Umsch 46: 429-434

2. Beisse R, Potulski M, Temme C et al. (1998) Das endoskopisch kontrollierte Zwerchfell-Splittting ein minimal invasiver Zugang zur ventralen Versorgung thorako-lumbaler Frakturen der Wirbelsäule. Unfallchirurg 101: 619-627

3. Beisse R, Potulski M, Bühren V (2001) Endoscopic techniques for the management of spinal trauma. Eur J Trauma 27: 275-291

4. Beisse R, Mueckley T, Schmidt MH et al. (2005) Surgical technique and results of endoscopic anterior spinal canal decompression. J Neurosurg 2: 128136

5. Dickman CA, Rosenthal DJ, Perin NI (eds) (1999) Thoracoscopic spine surgery. Thieme, Stuttgart New York

6. Faciszewski T, Winter RB, Lonstein JE et al. (1995) The surgical and medical perioperative complications of anterior spinal fusion. Surgery in the thoracic and lumbar spine in adults. Spine 20: 15921599

7. Khoo LT, Beisse R, Potulski M (2002) Thoracoscopic assisted treatment of thoracic and lumbar fractures: a series of 371 consecutive cases. Neurosurgery 51: 104-117

8. Kim DH, Jahng TA, Balabhadra RS et al. (2004) Thoracoscopic transdiaphragmatic approach to thoracolumbar junction fractures. Spine J 4: 317-328

9. Knop C, Bastian L, Lange U et al. (2002) Complications in surgical treatment of thoracolumbar injuries. Eur Spine J 11: 214-226

10. Muckley T, Schutz T, Schmidt MH et al. (2004) The role of thoracoscopic spinal surgery in the management of pyogenic vertebral osteomyelitis. Spine 29: E227-E233

11. Potulski M, Beisse R, Bühren V (1999) Die thorakoskopisch gesteuerte Behandlung der „Vorderen Säule". Orthopäde 28: 723-730

12. Regan JJ, Liebermann IH (eds) (2004) Atlas of minimal access spine surgery. Quality Medical Publishing, St Louis, p 560

13. Rosenthal D, Rosenthal R, Simone A (1994) Removal of a protruded disc using microsurgery endoscopy. Spine 19: 1087-1091 